\title{
Neonatal Outcome of Pregnancies Complicated with Idiopathic Thrombocytopenia
}

\author{
Surryia Zahid ${ }^{1,2}$, Raheela Asmat ${ }^{2}$, Naila Ehsan², Shakila Asmat ${ }^{2}$ and Tauseef M Asmat ${ }^{2}$ \\ ${ }^{1}$ Liaquat National Hospital, Karachi, Pakistan \\ ${ }^{2}$ Bolan Medical College, Quetta, Pakistan
}

\begin{abstract}
Objective: To determine the neonatal outcome with maternal idiopathic thrombocytopenia.

Study Design: Descriptive study.

Place and Duration of Study: Obstetrics and Gynecology Department, Liaqat National Hospital, Karachi, from January to June 2017.

Methodology: All 73 pregnant women diagnosed with idiopathic thrombocytopenia (ITP) during the study period were included. All the patients were kept under observation till birth of newborns. Neonates were assessed for thrombocytopenia, classified as mild, moderate and severe thrombocytopenia as outcome on the first post-natal day.

Results: Frequency of neonatal thrombocytopenia in 73 pregnant women complicated by idiopathic thrombocytopenia was $16.44 \%$ (12 neonates). Out of 12 cases, three (25\%) neonates had mild thrombocytopenia, four (33.33\%) neonates had moderate thrombocytopenia and five $(41.66 \%)$ neonates had severe thrombocytopenia.

Conclusion: There are considerable chances of neonatal thrombocytopenia when mothers were suffering with idiopathic thrombocytopenia during pregnancy. These findings may be useful for the medical staff to counsel pre-pregnant or pregnant women with ITP as well as care required during delivery.
\end{abstract}

Key Words: Thrombocytopenia, Idiopathic thrombocytopenia, Neonatal ITP, Pre-natal ITP.

How to cite this article: Zahid S, Asmat R, Ehsan N, Asmat S, Asmat TM. Neonatal Outcome of Pregnancies Complicated with Idiopathic Thrombocytopenia. J Coll Physicians Surg Pak 2020; 30(07):745-748.

\section{INTRODUCTION}

Thrombocytopenia is a complication of pregnancy and its occurrence has been reported in $10 \%$ of pregnant women worldwide. ${ }^{1,2}$ It is most common in young women during child-bearing age and may lead to several complications to mother and baby. ${ }^{3}$ Idiopathic thrombocytopenic purpura (ITP), also known as primary immune thrombocytopenic purpura, is an autoimmune disorder and greatcaution is desired to prevent the complications.

Low platelet count $\left(<150 * 10^{9}\right.$ per liter) is the main concern as it may cause coagulation abnormality in pregnancy and also may lead to secondary complications such as benign disorders, gestational thrombocytopenia and HELP syndrome with hemolysis and elevated liverenzymes. ${ }^{4}$

Low platelet count is a life-threatening condition for both mother and baby. ${ }^{5}$ Approximately $5 \%$ pregnant women in Pakistan suffer with mild thrombocytopenia at term without major sequelea. ${ }^{6}$

Correspondence to: Dr. Raheela Asmat, Bolan Medical

College Quetta, Pakistan

E-mail: asmatraheela@gmail.com

Received: January 16, 2018; Revised: July 12, 2019;

Accepted: December 26, 2019

DOI: https://doi.org/10.29271/jcpsp.2020.07.745
Although, the exact pathophysiology is unknown as to how this disease affects the fetus, it is assumed that the antibodies cross the placenta; thus, placing the infant at risk of thrombocytopenia. It is of paramount importance to treat such women with great care who has ITP during pregnancy, reducing the risk of hemorrhage so that both mother and baby can be saved. ITP in pregnancy carries high risk, and a vigilant eye is needed to observe ITP patients. ${ }^{8}$ In the past, studies have been conducted to observe the factors associated with the prognosis of newborn and mother with idiopathic thrombocytopenia pupura (ITP) and to compare the infants with and without thrombocytopenia in condition of maternal and neonatal characteristics. ${ }^{9}$ Another study conducted in Lahore, Pakistan during 2003 and 2004 reported the percentage of children admitted due to ITP, to be $4.8 \% .{ }^{10}$

Idiopathic thrombocytopenia in pregnant women has high potential to cause neonatal thrombocytopenia, which may further lead to adverse consequences. ${ }^{11}$ Thrombocytopenia in neonates from ITP pregnant women is underexplored in Pakistan. Therefore, this study was conducted to evaluate the frequency of neonatal thrombocytopenia in pregnant women complicated by idiopathic thrombocytopenia.

\section{METHODOLOGY}

Seventy-three pregnant women (sample size calculated using $p=10.3 \%$ and $d=7 \% n=73$ ) aged 18 to 35 years, diagnosed with 
Idiopathic thrombocytopenic at the Obstetrics and Gynecology Department, Liaqat National Hospital, Karachi, were included in this study. Patients were included on the basis of history, physical examination, and laboratory findings. Infant born to mothers with thrombocytopenia due to other causes such as systemic lupus erythematous (SLE) by anti-nuclear antibodies and anti-mitochondrial antibodies, pre-eclampsia, raised blood pressure $>140 / 90 \mathrm{~mm} \mathrm{Hg}$ after 20 weeks of gestation, proteinuria >300 mg/24 hours, hemolytic, hemolytic, anemia, elevated liver enzymes, and low platelet count (HELP) syndrome were excluded by lactate dehydrogenises, CBC platelet (HELP) count and liver function tests. The mother with sepsis history, temperature deranges, coagulation profile and serum fibrinogen level were also excluded. This study was conducted from January 2017 to June 2017. Only the women having low platelet counts $(<150,000 / \mu \mathrm{L})$ were included, while those with normal platelet count were taken as control in this study. All the patients were followed till delivery and newborns were assessed for thrombocytopenia according to operational definition, i.e. mild (platelet counts of 100,000 and $150,000 / \mu \mathrm{L}$ ), moderate (platelet counts 50,000 to $99,000 / \mu \mathrm{L}$ ) and severe thrombocytopenia (platelet counts $<50,000 / \mu \mathrm{L}$ ). Outcome was assessed on $1^{\text {st }}$ post-natal day by CBC platelet count.

A proforma was used to collect information from patients relived from labour room. An informed consent was obtained from the patients after informing them in simple and understandable language about the purpose of study, assuring them of confidentiality, and recognising their right to withdraw the consent at any time even without mentioning any reason for it.

Data was recorded and entered into SSPS Version 17 and analysed to see the effect modifiers like age, parity and gestational age. Descriptive statistics, given as the mean, were used for quantitative variables; while for frequencies and percentages, categorical variables were used. The level of significance (p) was taken at 0.05 .

\section{RESULTS}

The included 73 pregnant women diagnosed with idiopathic thrombocytopenia (ITP) during pregnancy had on average age of $25.64 \pm 3.84$ years and mean gestational age of the women were $38.30 \pm 1.27$ weeks. Twelve babies $(16.44 \%)$ of 73 neonates showed the signs and symptoms of ITP. Importantly, out of 12 newborns suffering with ITP, five suffered with severe thrombocytopenia while only three neonates had mild thrombocytopenia and four neonates suffered with moderate thrombocytopenia. Mostly, the women suffering with idiopathic thrombocytopenia were multiparous $(n=60)$, followed by primiparous $(n=13)$. The surprising outcome of results was that only one neonate suffered with ITP delivered by primiparous and 11 babies showed the signs of ITP delivered by multiparous mothers as shown in Tablel.

Out of the 73 mothers suffering with ITP, 42 were aged $<25$ years and delivered 8 neonates with the signs of ITP, while 23 women were of age between 26-30 and two babies suffered with ITP. The remaining 8 mothers were above 30 years of age and 2 newborns were delivered with signs and symptoms of ITP (Table I). These results indicate that chances of occurring neonatal thrombocytopenia is much higher in babies when delivered by young mothers suffering with ITP.

\section{DISCUSSION}

ITP is an autoimmune disorder, which is characterised by constant thrombocytopenia. ${ }^{12,13}$ The binding of antibody to platelet antigens, which leads to its premature destruction in the spleen is the primary etiology of ITP. ITP is defined by the American Society for Hematology guidelines as "isolated thrombocytopenia with no clinically apparent associated conditions or other causes of thrombocytopenia." Therefore, to diagnose ITP is critical and differential diagnosis from numerous other causes of thrombocytopenia, like hematological malignancies, medications, infections, disseminated intravascular coagulation and other autoimmune conditions should be performed. ${ }^{14,15}$

The findings of this study are in agreement with other reports published previously. ${ }^{16,17}$ van der Lugt et al., and Jahromi et al., reported up to $20 \%$ of infants suffering with ITP born to mothers diagnosed with ITP during pregnancy. ${ }^{16,17}$ Similarly, pregnancies complicated with ITP has been reported to have vaginal deliveries mostly (72.5\%), which is in agreement to current study. Importantly, 20 to $336^{10}$ per liters platelet counts in neonates have been reported by Melekoğlu et al. in $2017 .{ }^{7}$ Similar to our findings, none of the neonate had been reported to suffer with complication attributable to the mode of delivery. Moreover, the severity of ITP, mild in $10.3 \%$, moderate in $13.7 \%$ and severe in $24.1 \%$ neonates born to mothers suffering with ITP during pregnancies have been reported previously. ${ }^{7}$ The presently reported findings are also consistent with these reports.

However, chronic ITP (cITP) combined with ITP during pregnancy may have serious consequences and some studies have reported the complications and fetal death when mothers suffered with cITP during pregnancy. For example, the study conducted by Debouverie et al. examined 50 women suffering with CITP and had platelet counts below $150 \times 10^{9} / \mathrm{L}$ for minimum period of last one year. ${ }^{18}$ No fetal deaths in those pregnancies were reported, but 14\% were premature deliveries; while $9 \%$ neonates were small compared to their gestational age and importantly in 3\% evidence of hemorrhage were demonstrated. ${ }^{19}$

In this study, $16.44 \%$ neonates were born with neonatal thrombocytopenia, but none of those showed severe bleeding or intracranial hemorrhage, which is in agreement with previous reports. $^{20}$ However, some previously studies have also reported higher rates of severe neonatal thrombocytopenia. ${ }^{21}$ This study had the limitation that late neonatal outcomes were not studied. 
Table I: Frequency of neonatal thrombocytopenia in pregnant women with respect to maternal age and parity.

\begin{tabular}{|c|c|c|c|c|}
\hline \multirow{2}{*}{ Age groups (years) } & \multicolumn{2}{|c|}{ Neonatal thrombocytopenia } & \multirow{2}{*}{ Total } & \multirow{2}{*}{ p-value } \\
\hline & Yes & No & & \\
\hline$\leq 25$ years & $8(19 \%)$ & $34(81 \%)$ & 42 & \multirow{3}{*}{$\begin{array}{c}0.441 \\
(\text { Chi-square }=0.88)\end{array}$} \\
\hline 26 to 30 years & $2(8.7 \%)$ & $21(91.3 \%)$ & 23 & \\
\hline$>30$ years & $2(25 \%)$ & $6(75 \%)$ & 8 & \\
\hline Primiparous & $1(7.7 \%)$ & $12(92.3 \%)$ & 13 & \multirow{2}{*}{$\begin{array}{c}0.348 \\
(\text { Chi-square }=1.63)\end{array}$} \\
\hline Multiparous & $11(18.3 \%)$ & $49(81.7 \%)$ & 60 & \\
\hline
\end{tabular}

\section{CONCLUSION}

This study concludes that maternal ITP had no significant impairment of fetal well-being and all the cases of maternal ITP included in this study showed good maternal and neonatal outcomes. It is recommended that such pregnancies should be carefully monitored by an experienced team of obstetrician, hematologist, anesthesiologist, and neonatologist to minimise the risks.

\section{DISCLOSURE:}

The manuscript is the original study of the first author for her FCPS Part-II thesis work.

\section{ETHICAL APPROVAL:}

Ethical approvals were obtained prior to initiation of the research work from Hospital Ethical Committee to conduct the study.

\section{PATIENTS' CONSENTS:}

Informed consents were obtained from all patients to publish the data concerning this case.

\section{CONFLICT OF INTEREST:}

The authors declared no conflict of interest.

\section{AUTHORS' CONTRIBUTION:}

SZ: Data acquisition and analysis, interpretation, drafting and final approval.

NE, RA: Conception and design, Interpretation, critical revision and final approval.

SA, TMA: Critical revision and final approval.

\section{REFERENCES}

1. Gernsheimer T, James AH, Stasi R. How I treat thrombocytopenia in pregnancy. Blood 2013; 121(1):38-47.

2. Adams TM, Allaf MB, Vintzileos AM. Maternal thrombocytopenia in pregnancy: Diagnosis and management. Clin Lab Med 2013; 33(2):327-41.

3. Kong Z, Qin P, Xiao S, Zhou H, Li H, Yang R, et al. A novel recombinant human thrombopoietin therapy for the management of immune thrombocytopenia in pregnancy. Blood 2017; 130(9):1097-1103.

4. Loustou V, Debouverie O, Canoui Poitrine F. Effect of pregnancy on the course of immune thrombocytopenia: a retrospective study of 118 pregnancies in 52 women. $\mathrm{Br}$ J Haematol 2014; 166(6):929-35.

5. Hachisuga K, Hidaka N, Fujita Y, Fukushima K, Kato K. Can we predict neonatal thrombocytopenia in offspring of women with idiopathic thrombocytopenic purpura. Blood Res 2014; 49(4):259-64.

6. Alam M, Ansari A. Asymptomatic thrombocytopenic at term pregnancy. Pak J Pathol 2007; 18(1):17-9.

7. Melekoğlu NA, Bay A, Aktekin EH, Yilmaz M, Sivasli E. Neonatal outcomes of pregnancy with immune thrombocytopenia. Ind J Hem Blood Tran 2017; 33(2):211-15.

8. Bayhan T, Tavil B, Korkmaz A, Ünal Ş, Hanalioğlu D, Yiğit Ş, et al. Neonates born to mothers with immune thrombocytopenic purpura: A single-center experience of 20 years. Blood Coagul Fibrinolysis 2016; 27(1):19-23.

9. Ozkan M, Cetinkaya M, Kokusai N, Ali R. Neonatal outcome of pregnancy complicated by idiopathic thrombocytopenia purpura. J Perinatal 2010; 30:38-44.

10. Ali AS. Idiopathic thrombocytopenic purpura-initial illness and subsequent behavior. Pak Pediatr J 2005; 29(2):93-9.

11. Stavrou E, McCrae KR. Immune thrombocytopenia in pregnancy. Hematol Oncol Clin North Am 2009; 23(6): 1299-316.

12. Kamphuis MM, Paridaans NP, Porcelijn L, Lopriore E, Oepkes D. Incidence and consequences of neonatal alloimmune thrombocytopenia: A systematic review. Pediatrics 2014; 133(4):715-21.

13. von Auer C, von Krogh AS, Kremer Hovinga JA, Lämmle B. Current insights into thrombotic microangiopathies: Thrombotic thrombocytopenic purpura and pregnancy. Thromb Res 2015; 135(Suppl1):30-3.

14. Scully M, Thomas M, Underwood M, Watson H, Langley $K$, Camilleri RS, et al. Thrombotic thrombocytopenic purpura and pregnancy: Presentation, management, and subsequent pregnancy outcomes. Blood 2014; 124(2): 211-9.

15. Noris P, Schlegel N, Kiersy C, PujolMoix N. Analysis of 339 pregnancies in 181 women with 13 different forms of inherited thrombocytopenia. Haematologica 2014; 99(8): 1387-94.

16. Van der Lugt NM, van Kampen A, Walther FJ, Brand A, Lopriore E. Outcome and management in neonatal thrombocytopenia due to maternal idiopathic thrombocytopenic purpura. Vox Sang 2013; 105(3): 236-43.

17. Namavar Jahromi B, Shiravani Z, Salarian L. Perinatal outcome of pregnancies complicated by immune 
thrombocytopenia. Iran Red Crescent Med J 2012; 14(7): 430-5.

18. Debouverie O, Roblot P, Roy-Péaud F, Boinot C, Pierre F, and Pourrat $O$. Chronic idiopathic thrombocytopenia outcome during pregnancy (62 cases). Revue de Med Interne 2012; 33(8):426-32.

19. Subbaiah M, Kumar S, Roy KK, Sharma JB, Singh N. Pregnancyoutcome in patients with idiopathic thrombocytopenic purpura. Arch Gynecol Obstet 2014; 289(2):
269-73.

20. Wyszynski DF, Carman WJ, Cantor AB, Graham JM, Kunz LH, Slavotinek AM, et al. Pregnancy and birth outcomes among women with idiopathic thrombocytopenic purpura. J Pregnancy 2016; 2016:8297407.

21. Ree IMC, FustoloGunnink SF, Bekker V, Fijnvandraat KJ, Steggerda SJ, Lopriore E. Thrombocytopenia in neonatal sepsis: Incidence, severity and risk factors. Simeoni U, ed. PLoS One 2017; 12(10): 0185581. 